\title{
Supporting Information: \\ Impact of Different Automated Binding Pose Generation Approaches on Relative Binding Free Energy Simulations
}

\author{
Daniel Cappel, ${ }^{*, \dagger}$ Steven Jerome, ${ }^{\ddagger}$ Gerhard Hessler, $₫$ and Hans Matter, ${ }^{*}$ \\ $\dagger$ †chrödinger GmbH, 68161 Mannheim, Germany \\ $\ddagger$ Schrödinger Inc., New York, New York 10036, United States \\ IIntegrated Drug Discovery (IDD), Synthetic Molecular Design, Sanofi-Aventis \\ Deutschland GmbH, 65926 Frankfurt am Main, Germany \\ E-mail: daniel.cappel@schrodinger.com; hans.matter@sanofi.com
}



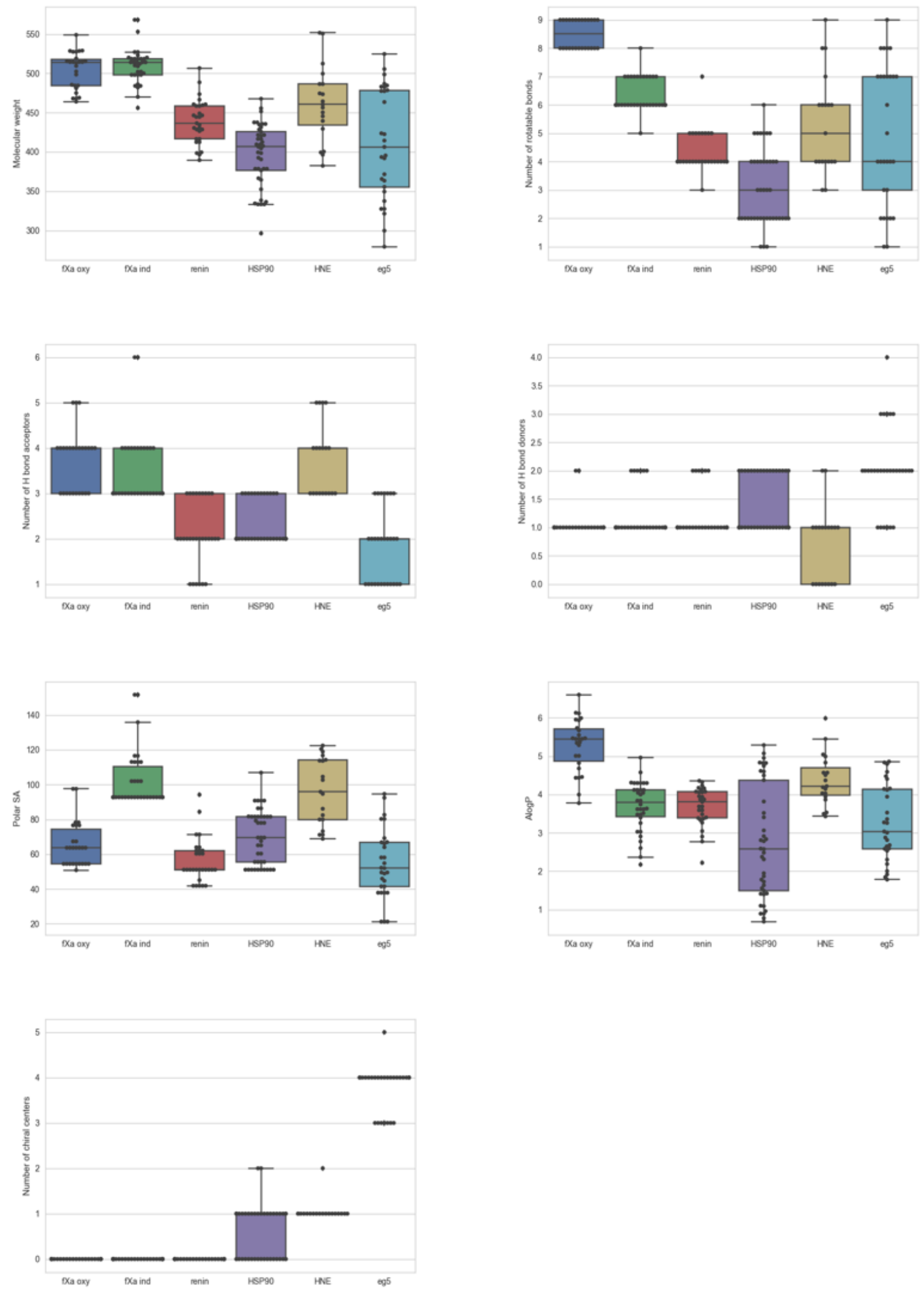

Figure S1: Box plots shown together with property values for different metrics characterising studied ligand series. 
Table S1: Experimental Binding Affinity and FEP+ Predictions for the fXa Oxybenzamide Data Set. ${ }^{a}$

\begin{tabular}{lrrrrr}
\hline Ligand & $\Delta G_{\exp }$ & $\Delta G_{\text {mcsdocking }}$ & $\Delta G_{\text {XPcore05 }}$ & $\Delta G_{\text {XPcore10 }}$ & $\Delta G_{\text {flex_mcs }}$ \\
\hline jmc_1 & -10.50 & $-10.78 \pm 0.13$ & $-11.69 \pm 0.13$ & $-11.55 \pm 0.28$ & $-11.05 \pm 0.13$ \\
jmc_2 & -9.96 & $-10.09 \pm 0.15$ & $-11.00 \pm 0.13$ & $-10.66 \pm 0.32$ & $-10.29 \pm 0.12$ \\
jmc_4 & -10.14 & $-10.21 \pm 0.21$ & $-5.71 \pm 0.13$ & $-10.72 \pm 0.31$ & $-10.26 \pm 0.13$ \\
jmc_5 & -10.16 & $-8.61 \pm 0.14$ & $-9.19 \pm 0.18$ & $-9.01 \pm 0.27$ & $-9.01 \pm 0.14$ \\
jmc_6 & -9.54 & $-8.06 \pm 0.15$ & $-7.80 \pm 0.14$ & $-7.73 \pm 0.26$ & $-7.67 \pm 0.14$ \\
jmc_7 & -9.42 & $-8.75 \pm 0.14$ & $-7.53 \pm 0.18$ & $-8.37 \pm 0.35$ & $-8.39 \pm 0.14$ \\
jmc_9 & -7.92 & $-6.77 \pm 0.16$ & $-7.07 \pm 0.14$ & $-6.96 \pm 0.37$ & $-6.78 \pm 0.14$ \\
jmc_10 & -7.80 & $-8.59 \pm 0.17$ & $-6.52 \pm 0.13$ & $-6.86 \pm 0.27$ & $-8.77 \pm 0.13$ \\
jmc_20 & -8.39 & $-8.00 \pm 0.15$ & $-5.81 \pm 0.17$ & $-6.71 \pm 0.29$ & $-7.60 \pm 0.17$ \\
jmc_25 & -9.52 & $-9.65 \pm 0.16$ & $-10.36 \pm 0.16$ & $-10.37 \pm 0.26$ & $-9.79 \pm 0.13$ \\
jmc_28 & -10.14 & $-10.58 \pm 0.15$ & $-11.18 \pm 0.13$ & $-11.44 \pm 0.25$ & $-10.66 \pm 0.13$ \\
jmc_30 & -10.04 & $-10.46 \pm 0.15$ & $-11.28 \pm 0.18$ & $-11.43 \pm 0.35$ & $-10.79 \pm 0.13$ \\
jmc_36 & -8.50 & $-8.57 \pm 0.15$ & $-9.09 \pm 0.13$ & $-9.60 \pm 0.26$ & $-8.82 \pm 0.13$ \\
jmc_37 & -9.95 & $-10.73 \pm 0.17$ & $-11.13 \pm 0.13$ & $-11.40 \pm 0.28$ & $-10.54 \pm 0.13$ \\
jmc_41 & -9.42 & $-10.26 \pm 0.17$ & $-10.52 \pm 0.14$ & $-10.64 \pm 0.33$ & $-9.89 \pm 0.14$ \\
jmc_48 & -10.91 & $-11.12 \pm 0.15$ & $-11.68 \pm 0.13$ & $-11.41 \pm 0.25$ & $-10.39 \pm 0.14$ \\
jmc_49 & -10.76 & $-11.13 \pm 0.13$ & $-11.61 \pm 0.13$ & $-11.27 \pm 0.26$ & $-11.18 \pm 0.13$ \\
jmc_50 & -10.57 & $-8.83 \pm 0.16$ & $-9.52 \pm 0.14$ & $-9.42 \pm 0.25$ & $-9.11 \pm 0.12$ \\
jmc_52 & -9.92 & $-9.05 \pm 0.16$ & $-9.18 \pm 0.17$ & $-5.81 \pm 0.30$ & $-8.78 \pm 0.23$ \\
jmc_53 & -10.08 & $-10.88 \pm 0.13$ & $-11.48 \pm 0.12$ & $-11.40 \pm 0.25$ & $-10.74 \pm 0.14$ \\
jmc_56 & -10.37 & $-8.86 \pm 0.19$ & $-8.88 \pm 0.13$ & $-8.71 \pm 0.26$ & $-8.42 \pm 0.13$ \\
jmc_59 & -9.88 & $-9.05 \pm 0.14$ & $-9.50 \pm 0.17$ & $-9.65 \pm 0.31$ & $-9.55 \pm 0.15$ \\
jmc_60 & -9.49 & $-10.34 \pm 0.15$ & $-10.96 \pm 0.16$ & $-8.06 \pm 0.34$ & $-10.20 \pm 0.14$ \\
jmc_84 & -6.82 & $-8.62 \pm 0.14$ & $-8.82 \pm 0.13$ & $-8.50 \pm 0.30$ & $-8.67 \pm 0.17$ \\
jmc_88 & -6.82 & $-7.40 \pm 0.13$ & $-7.73 \pm 0.17$ & $-7.42 \pm 0.26$ & $-7.75 \pm 0.13$ \\
jmc_96 & -7.53 & $-9.16 \pm 0.19$ & $-9.29 \pm 0.14$ & $-9.42 \pm 0.25$ & $-9.42 \pm 0.14$ \\
\hline aA1 $\Delta G$ & va & &
\end{tabular}

${ }^{a}$ All $\Delta G$ values and predicted errors are given in $\mathrm{kcal} \cdot \mathrm{mol}^{-1}$. 
Table S2: Experimental Binding Affinity and FEP+ Predictions for the fXa Oxybenzamide Data Set with 20 ns Simulation Time per $\lambda$ Window. $^{a}$

\begin{tabular}{lrrr}
\hline Ligand & $\Delta G_{\text {exp }}$ & $\Delta G_{\text {mcsdocking }}$ & $\Delta G_{\text {XPcore10 }}$ \\
\hline jmc_1 & -10.50 & $-10.23 \pm 0.11$ & $-11.28 \pm 0.17$ \\
jmc_2 & -9.96 & $-10.00 \pm 0.12$ & $-10.46 \pm 0.18$ \\
jmc_4 & -10.14 & $-10.01 \pm 0.13$ & $-10.18 \pm 0.24$ \\
jmc_5 & -10.16 & $-8.87 \pm 0.14$ & $-9.15 \pm 0.17$ \\
jmc_6 & -9.54 & $-7.87 \pm 0.14$ & $-7.68 \pm 0.17$ \\
jmc_7 & -9.42 & $-8.12 \pm 0.16$ & $-7.92 \pm 0.24$ \\
jmc_9 & -7.92 & $-7.56 \pm 0.13$ & $-6.98 \pm 0.29$ \\
jmc_10 & -7.80 & $-8.20 \pm 0.14$ & $-7.12 \pm 0.18$ \\
jmc_20 & -8.39 & $-7.80 \pm 0.15$ & $-7.00 \pm 0.16$ \\
jmc_25 & -9.52 & $-9.62 \pm 0.11$ & $-10.27 \pm 0.17$ \\
jmc_28 & -10.14 & $-10.39 \pm 0.11$ & $-11.15 \pm 0.16$ \\
jmc_30 & -10.04 & $-10.62 \pm 0.13$ & $-11.01 \pm 0.25$ \\
jmc_36 & -8.50 & $-9.18 \pm 0.16$ & $-9.28 \pm 0.16$ \\
jmc_37 & -9.95 & $-10.30 \pm 0.13$ & $-11.15 \pm 0.16$ \\
jmc_41 & -9.42 & $-9.66 \pm 0.12$ & $-10.00 \pm 0.24$ \\
jmc_48 & -10.91 & $-10.93 \pm 0.13$ & $-11.30 \pm 0.16$ \\
jmc_49 & -10.76 & $-10.83 \pm 0.13$ & $-11.24 \pm 0.16$ \\
jmc_50 & -10.57 & $-9.36 \pm 0.13$ & $-9.40 \pm 0.16$ \\
jmc_52 & -9.92 & $-9.19 \pm 0.12$ & $-7.74 \pm 0.20$ \\
jmc_53 & -10.08 & $-10.76 \pm 0.11$ & $-11.12 \pm 0.16$ \\
jmc_56 & -10.37 & $-8.81 \pm 0.13$ & $-8.70 \pm 0.16$ \\
jmc_59 & -9.88 & $-9.47 \pm 0.11$ & $-9.38 \pm 0.22$ \\
jmc_60 & -9.49 & $-10.19 \pm 0.11$ & $-9.56 \pm 0.19$ \\
jmc_84 & -6.82 & $-9.09 \pm 0.11$ & $-8.50 \pm 0.19$ \\
jmc_88 & -6.82 & $-7.98 \pm 0.11$ & $-7.88 \pm 0.16$ \\
jmc_96 & -7.53 & $-9.48 \pm 0.13$ & $-9.08 \pm 0.16$ \\
\hline $0 \mathrm{AII} G$ & &
\end{tabular}

${ }^{a}$ All $\Delta G$ values and predicted errors are given in $\mathrm{kcal} \cdot \mathrm{mol}^{-1}$. 
Table S3: Experimental Binding Affinity and FEP+ Predictions for the fXa Indole Data Set. ${ }^{a}$

\begin{tabular}{|c|c|c|c|c|c|}
\hline Ligand & $\Delta G_{\exp }$ & $\Delta G_{\text {mcsdocking }}$ & $\Delta G_{\text {XPcore05 }}$ & $\Delta G_{\text {XPcore10 }}$ & $\Delta G_{\text {flex }_{-}}$ \\
\hline CPD_01 & -13.64 & $-12.78 \pm 0.11$ & $-12.40 \pm 0.24$ & $-9.51 \pm 0.13$ & $-12.41 \pm 0.16$ \\
\hline CPD_02 & -13.11 & $-11.07 \pm 0.13$ & $-11.73 \pm 0.23$ & $-11.68 \pm 0.14$ & $-11.46 \pm 0.13$ \\
\hline CPD_03 & -13.03 & $-12.61 \pm 0.12$ & $-12.18 \pm 0.24$ & $-12.73 \pm 0.14$ & $-12.33 \pm 0.16$ \\
\hline CPD_04 & -12.31 & $-11.17 \pm 0.11$ & $-11.33 \pm 0.24$ & $-11.19 \pm 0.14$ & $-11.19 \pm 0.10$ \\
\hline CPD_05 & -11.87 & $-11.88 \pm 0.13$ & $-11.82 \pm 0.24$ & $-12.30 \pm 0.17$ & $-11.88 \pm 0.15$ \\
\hline CPD_06 & -11.75 & $-12.16 \pm 0.11$ & $-11.91 \pm 0.22$ & $-13.01 \pm 0.15$ & $-12.07 \pm 0.10$ \\
\hline CPD_07 & -11.66 & $-11.55 \pm 0.11$ & $-8.69 \pm 0.23$ & $-8.38 \pm 0.15$ & $-11.28 \pm 0.10$ \\
\hline CPD_08 & -11.65 & $-10.68 \pm 0.12$ & $-10.36 \pm 0.25$ & $-10.93 \pm 0.16$ & $-10.32 \pm 0.10$ \\
\hline CPD_09_Xray & -11.55 & $-11.61 \pm 0.12$ & $-11.47 \pm 0.23$ & $-11.54 \pm 0.13$ & $-11.53 \pm 0.10$ \\
\hline CPD_10 & -11.54 & $-10.87 \pm 0.12$ & $-11.37 \pm 0.23$ & $-11.48 \pm 0.17$ & $-11.10 \pm 0.10$ \\
\hline CPD_11 & -11.54 & $-11.90 \pm 0.13$ & -12.5 & $-12.48 \pm 0.13$ & $-12.05 \pm 0.12$ \\
\hline CPD_12 & -11.51 & $-10.36 \pm 0.12$ & $-10.73 \pm 0.23$ & $-10.51 \pm 0.14$ & $-10.16 \pm 0.11$ \\
\hline CPD_13 & -11.51 & $-9.47 \pm$ & $-11.03 \pm 0.24$ & $-9.91=$ & -9.58 \\
\hline CPD_14 & & $-10.72 \pm 0.12$ & $-11.18 \pm 0.23$ & $-11.48 \pm 0.14$ & $-11.27 \pm 0.10$ \\
\hline CPD_15 & -11.38 & $-10.73 \pm 0.12$ & $-9.92 \pm 0.28$ & $-11.76 \pm 0.16$ & $-10.75 \pm 0.10$ \\
\hline CPD_16 & -11.27 & $-10.80 \pm 0.12$ & $-10.52 \pm 0.22$ & $-10.74 \pm 0.14$ & $-10.97 \pm 0.16$ \\
\hline CPD_17 & & $-10.89 \pm 0.11$ & $-11.04 \pm 0.23$ & $-10.83 \pm 0.13$ & $-10.70 \pm 0.10$ \\
\hline CPD_18 & -11.13 & $-12.49 \pm 0.11$ & $-12.47 \pm 0.24$ & $-12.06 \pm 0.13$ & $-11.81 \pm 0.10$ \\
\hline CPD_19 & & $-11.51 \pm 0.12$ & $-11.34 \pm 0.25$ & $-12.36 \pm 0.13$ & $-11.49 \pm 0.10$ \\
\hline CPD_20 & -11.10 & $-11.60 \pm 0.11$ & $-11.77 \pm 0.24$ & $-12.77 \pm 0.16$ & $-11.16 \pm 0.10$ \\
\hline CPD_21 & -11.09 & $-11.80 \pm 0.11$ & $-11.63 \pm 0.25$ & $-11.23 \pm 0.15$ & $-11.33 \pm 0.10$ \\
\hline CPD_22 & & $-10.53 \pm 0.12$ & $-10.46 \pm 0.22$ & $-11.17 \pm 0.15$ & $-10.63 \pm 0.11$ \\
\hline CPD_23 & -10.76 & $-10.61 \pm 0.11$ & $-10.88 \pm 0.21$ & $-10.83 \pm 0.14$ & $-10.39 \pm 0.11$ \\
\hline CPD_24 & -10.68 & $-10.82 \pm 0.15$ & $-11.36 \pm 0.22$ & $-7.73 \pm 0.15$ & $-11.20 \pm 0.11$ \\
\hline CPD_25 & -10.67 & $-10.34 \pm 0.18$ & $-11.05 \pm 0.25$ & $-11.26 \pm 0.13$ & $-10.96 \pm 0.11$ \\
\hline CPD_26 & -10.58 & $-10.68 \pm 0.12$ & $-11.17 \pm 0.24$ & $-11.48 \pm 0.17$ & $-10.89 \pm 0.10$ \\
\hline CPD_27 & -10.56 & $-11.89 \pm 0.11$ & $-11.51 \pm 0.25$ & $-11.92 \pm 0.13$ & $-11.31 \pm 0.10$ \\
\hline CPD_28 & -10.23 & $-11.12 \pm 0.12$ & $-11.40 \pm 0.26$ & $-11.42 \pm 0.13$ & $-11.27 \pm 0.11$ \\
\hline CPD_29 & -10.09 & $-9.98 \pm 0.16$ & $-10.15 \pm 0.24$ & $-7.55 \pm 0.17$ & $-10.08 \pm 0.10$ \\
\hline CPD_30 & -9.75 & $-11.23 \pm 0.13$ & $-10.65 \pm 0.22$ & $-11.60 \pm 0.15$ & $-10.89 \pm 0.11$ \\
\hline CPD_31 & -9.39 & $-9.92 \pm 0.13$ & $-10.54 \pm 0.20$ & $-10.71 \pm 0.16$ & $-10.50 \pm 0.10$ \\
\hline CPD_32 & -9.35 & $-10.32 \pm 0.12$ & $-10.60 \pm 0.22$ & $-11.30 \pm 0.15$ & $-10.74 \pm 0.11$ \\
\hline CPD_33 & -9.09 & $-10.22 \pm 0.12$ & $-10.20 \pm 0.22$ & $-10.55 \pm 0.13$ & $-10.25 \pm 0.10$ \\
\hline CPD_34 & -7.74 & $-10.08 \pm 0.18$ & $-8.96 \pm 0.26$ & $-9.96 \pm 0.15$ & $-10.43 \pm 0.18$ \\
\hline
\end{tabular}

${ }^{a}$ All $\Delta G$ values and predicted errors are given in $\mathrm{kcal} \cdot \mathrm{mol}^{-1}$. 
Table S4: Experimental Binding Affinity and FEP+ Predictions for the fXa Indole Data Set with 20 ns Simulation Time per $\lambda$ Window. ${ }^{a}$

\begin{tabular}{lrr}
\hline Ligand & $\Delta G_{\text {exp }}$ & $\Delta G_{\text {XPcore0 }}$ \\
\hline CPD_01 & -13.64 & $-11.89 \pm 0.13$ \\
CPD_02 & -13.11 & $-11.18 \pm 0.15$ \\
CPD_03 & -13.03 & $-12.19 \pm 0.14$ \\
CPD_04 & -12.31 & $-10.76 \pm 0.14$ \\
CPD_05 & -11.87 & $-12.30 \pm 0.15$ \\
CPD_06 & -11.75 & $-11.95 \pm 0.14$ \\
CPD_07 & -11.66 & $-9.19 \pm 0.16$ \\
CPD_08 & -11.65 & $-10.44 \pm 0.14$ \\
CPD_09_Xray & -11.55 & $-11.67 \pm 0.14$ \\
CPD_10 & -11.54 & $-11.02 \pm 0.15$ \\
CPD_11 & -11.54 & $-12.18 \pm 0.13$ \\
CPD_12 & -11.51 & $-10.44 \pm 0.14$ \\
CPD_13 & -11.51 & $-10.07 \pm 0.14$ \\
CPD_14 & -11.48 & $-11.19 \pm 0.13$ \\
CPD_15 & -11.38 & $-10.80 \pm 0.18$ \\
CPD_16 & -11.27 & $-10.50 \pm 0.16$ \\
CPD_17 & -11.24 & $-11.28 \pm 0.14$ \\
CPD_18 & -11.13 & $-11.93 \pm 0.13$ \\
CPD_19 & -11.13 & $-11.86 \pm 0.16$ \\
CPD_20 & -11.10 & $-11.73 \pm 0.14$ \\
CPD_21 & -11.09 & $-11.85 \pm 0.14$ \\
CPD_22 & -10.98 & $-10.55 \pm 0.15$ \\
CPD_23 & -10.76 & $-10.58 \pm 0.13$ \\
CPD_24 & -10.68 & $-11.26 \pm 0.13$ \\
CPD_25 & -10.67 & $-11.15 \pm 0.14$ \\
CPD_26 & -10.58 & $-10.79 \pm 0.13$ \\
CPD_27 & -10.55 & $-11.93 \pm 0.16$ \\
CPD_28 & -10.23 & $-10.98 \pm 0.14$ \\
CPD_29 & -10.09 & $-10.04 \pm 0.14$ \\
CPD_30 & -9.75 & $-10.92 \pm 0.16$ \\
CPD_31 & -9.39 & $-10.43 \pm 0.13$ \\
CPD_32 & -9.35 & $-10.56 \pm 0.14$ \\
CPD_33 & -9.09 & $-10.50 \pm 0.14$ \\
CPD_34 & -7.74 & $-10.29 \pm 0.17$ \\
\hline aAl $\Delta G$ values and predicted errors $a r e$ \\
given in kcal $\cdot$ mol -1. & \\
\hline & &
\end{tabular}


Table S5: Experimental Binding Affinity and FEP+ Predictions for the Renin Data Set. ${ }^{a}$

\begin{tabular}{|c|c|c|c|c|c|}
\hline Ligand & $\Delta G_{\exp }$ & $\Delta G_{\text {mcsdocking }}$ & $\Delta G_{\text {XPcore05 }}$ & $\Delta G_{\text {XPcore } 10}$ & $\Delta G_{\text {flex } \_ \text {mcs }}$ \\
\hline $\mathrm{b} 1 \mathrm{a} 2 \mathrm{~m}$ & -9.61 & $-10.69 \pm 0.16$ & $-11.03 \pm 0.22$ & $-10.31 \pm 0.16$ & $-10.87 \pm 0.16$ \\
\hline b1i1 & -7.39 & $-8.25 \pm 0.27$ & $-8.06 \pm 0.25$ & $-8.04 \pm 0.17$ & $-6.70 \pm 0.16$ \\
\hline b1i2a & -8.70 & $-9.78 \pm 0.16$ & $-9.32 \pm 0.23$ & $-9.56 \pm 0.17$ & $-9.36 \pm 0.22$ \\
\hline b1i2c & -6.88 & $-6.14 \pm 0.16$ & $-6.31 \pm 0.29$ & $-6.75 \pm 0.17$ & $-6.23 \pm 0.16$ \\
\hline b1i2d & -8.41 & $-7.81 \pm 0.16$ & $-7.29 \pm 0.25$ & $-8.06 \pm 0.15$ & $-8.33 \pm 0.29$ \\
\hline b1i2e & -10.47 & $-10.29 \pm 0.16$ & $-10.69 \pm 0.26$ & $-9.91 \pm 0.23$ & $-10.43 \pm 0.19$ \\
\hline b1i2g & -8.34 & $-8.61 \pm 0.18$ & $-9.03 \pm 0.24$ & $-8.81 \pm 0.18$ & $-9.41 \pm 0.16$ \\
\hline b1i2h & -9.82 & $-9.79 \pm 0.16$ & $-8.48 \pm 0.26$ & $-8.85 \pm 0.17$ & $-9.84 \pm 0.17$ \\
\hline b1i2i & -9.81 & $-10.91 \pm 0.17$ & $-10.87 \pm 0.29$ & $-10.85 \pm 0.16$ & $-11.14 \pm 0.15$ \\
\hline $\mathrm{b} 1 \mathrm{i} 2 \mathrm{j}$ & -11.32 & $-10.52 \pm 0.17$ & $-10.10 \pm 0.25$ & $-10.19 \pm 0.21$ & $-8.67 \pm 0.15$ \\
\hline b1i2k & -10.64 & $-10.76 \pm 0.16$ & $-10.63 \pm 0.22$ & $-10.60 \pm 0.15$ & $-9.85 \pm 0.15$ \\
\hline b1i2n & -10.98 & $-10.36 \pm 0.24$ & $-10.97 \pm 0.29$ & $-10.74 \pm 0.16$ & $-11.87 \pm 0.19$ \\
\hline b1i2o & -10.57 & $-10.67 \pm 0.18$ & $-11.15 \pm 0.30$ & $-11.69 \pm 0.17$ & $-11.47 \pm 0.15$ \\
\hline b1i4a & -7.24 & $-8.49 \pm 0.16$ & $-8.51 \pm 0.22$ & $-8.06 \pm 0.16$ & $-8.28 \pm 0.15$ \\
\hline b1i4b & -8.39 & $-9.45 \pm 0.17$ & $-8.70 \pm 0.26$ & $-9.29 \pm 0.15$ & $-9.54 \pm 0.16$ \\
\hline b1i4e & -10.19 & $-9.45 \pm 0.16$ & $-10.96 \pm 0.25$ & $-10.91 \pm 0.18$ & $-9.73 \pm 0.16$ \\
\hline b1i4g & -8.35 & $-7.24 \pm 0.16$ & $-8.04 \pm 0.23$ & $-7.81 \pm 0.16$ & $-8.14 \pm 0.19$ \\
\hline b1i4i & -8.79 & $-8.68 \pm 0.27$ & $-8.99 \pm 0.22$ & $-11.51 \pm 0.17$ & $-10.00 \pm 0.20$ \\
\hline b1i5a & -10.40 & $-9.84 \pm 0.16$ & $-9.63 \pm 0.31$ & $-9.12 \pm 0.17$ & $-8.57 \pm 0.15$ \\
\hline b1i5b & -8.01 & $-10.13 \pm 0.17$ & $-9.47 \pm 0.27$ & $-9.70 \pm 0.17$ & $-7.68 \pm 0.16$ \\
\hline b1i5c & -11.87 & $-10.34 \pm 0.17$ & $-10.07 \pm 0.25$ & $-10.51 \pm 0.17$ & $-8.83 \pm 0.17$ \\
\hline b1i5d & -11.22 & $-10.80 \pm 0.17$ & $-11.32 \pm 0.29$ & $-11.16 \pm 0.18$ & $-9.05 \pm 0.16$ \\
\hline b1i5h & -10.81 & $-10.50 \pm 0.19$ & $-9.54 \pm 0.27$ & $-10.28 \pm 0.17$ & $-10.96 \pm 0.16$ \\
\hline b1i5i & -11.46 & $-11.22 \pm 0.22$ & $-10.60 \pm 0.28$ & $-10.12 \pm 0.16$ & $-12.36 \pm 0.18$ \\
\hline b1i5j & -11.46 & $-10.73 \pm 0.17$ & $-11.10 \pm 0.22$ & $-10.86 \pm 0.17$ & $-11.61 \pm 0.16$ \\
\hline b1i5k_xray & -11.87 & $-10.97 \pm 0.18$ & $-10.37 \pm 0.26$ & $-10.19 \pm 0.18$ & $-11.46 \pm 0.15$ \\
\hline b1i5l & -9.65 & $-10.01 \pm 0.19$ & $-9.75 \pm 0.28$ & $-9.63 \pm 0.16$ & $-10.57 \pm 0.15$ \\
\hline b1i5n & -10.24 & $-10.13 \pm 0.20$ & $-10.57 \pm 0.29$ & $-9.35 \pm 0.17$ & $-10.57 \pm 0.16$ \\
\hline b1i5o & -10.04 & $-10.38 \pm 0.26$ & $-11.35 \pm 0.32$ & $-10.04 \pm 0.23$ & $-11.41 \pm 0.27$ \\
\hline
\end{tabular}

${ }^{a}$ All $\Delta G$ values and predicted errors are given in $\mathrm{kcal} \cdot \mathrm{mol}^{-1}$. 
Table S6: Experimental Binding Affinity and FEP+ Predictions for the Renin Data Set with 20 ns Simulation Time per $\lambda$ Window. ${ }^{a}$

\begin{tabular}{lrr}
\hline Ligand & $\Delta G_{\text {exp }}$ & $\Delta G_{\text {XPcore10 }}$ \\
\hline b1a2m & -9.61 & $-10.15 \pm 0.11$ \\
b1i1 & -7.39 & $-8.38 \pm 0.11$ \\
b1i2a & -8.70 & $-9.17 \pm 0.11$ \\
b1i2c & -6.88 & $-5.92 \pm 0.12$ \\
b1i2d & -8.41 & $-7.98 \pm 0.11$ \\
b1i2e & -10.47 & $-9.77 \pm 0.13$ \\
b1i2g & -8.34 & $-8.87 \pm 0.11$ \\
b1i2h & -9.82 & $-9.14 \pm 0.12$ \\
b1i2i & -9.81 & $-10.70 \pm 0.11$ \\
b1i2j & -11.32 & $-10.50 \pm 0.12$ \\
b1i2k & -10.64 & $-10.03 \pm 0.11$ \\
b1i2n & -10.98 & $-11.05 \pm 0.13$ \\
b1i2o & -10.57 & $-11.53 \pm 0.13$ \\
b1i4a & -7.24 & $-8.32 \pm 0.11$ \\
b1i4b & -8.39 & $-8.93 \pm 0.11$ \\
b1i4e & -10.19 & $-11.12 \pm 0.12$ \\
b1i4g & -8.35 & $-8.44 \pm 0.11$ \\
b1i4i & -8.79 & $-11.70 \pm 0.13$ \\
b1i5a & -10.40 & $-10.37 \pm 0.13$ \\
b1i5b & -8.01 & $-9.56 \pm 0.13$ \\
b1i5c & -11.87 & $-9.76 \pm 0.13$ \\
b1i5d & -11.22 & $-10.37 \pm 0.12$ \\
b1i5h & -10.81 & $-10.96 \pm 0.12$ \\
b1i5i & -11.46 & $-10.26 \pm 0.11$ \\
b1i5j & -11.46 & $-10.57 \pm 0.14$ \\
b1i5k_xray & -11.87 & $-10.60 \pm 0.12$ \\
b1i5l & -9.65 & $-9.95 \pm 0.11$ \\
b1i5n & -10.24 & $-9.37 \pm 0.13$ \\
b1i5o & -10.04 & $-9.44 \pm 0.12$ \\
\hline aA11 $\Delta G$ values and predicted errors are \\
b1
\end{tabular}

${ }^{a}$ All $\Delta G$ values and predicted errors are given in $\mathrm{kcal} \cdot \mathrm{mol}^{-1}$. 
Table S7: Experimental Binding Affinity and FEP+ Predictions for the HSP90 Data Set. ${ }^{a}$

\begin{tabular}{|c|c|c|c|c|c|}
\hline Ligand & $\Delta G_{\exp }$ & $\Delta G_{\text {mcsdocking }}$ & $\Delta G_{\text {SPcore } 01}$ & $\Delta G_{\text {flex_scaf }}$ & $\Delta G_{\text {flex_mcs }}$ \\
\hline 1 & -11.63 & $-12.02 \pm 0.25$ & $-12.85 \pm 0.18$ & $-14.02 \pm 0.34$ & $-13.14 \pm 0.13$ \\
\hline 3 & -9.06 & $-6.35 \pm 0.25$ & $-7.39 \pm 0.18$ & $-8.08 \pm 0.33$ & $-7.21 \pm 0.13$ \\
\hline $4-0$ & $>-6.82$ & $-4.57 \pm 1.27$ & $-6.20 \pm 0.27$ & $-4.05 \pm 2.34$ & $-6.25 \pm 0.28$ \\
\hline $4-1$ & $>-6.82$ & & $-1.73 \pm 0.71$ & & \\
\hline 5 & $>-6.82$ & $-6.94 \pm 1.40$ & $-8.76 \pm 0.97$ & $-9.73 \pm 0.37$ & $-8.94 \pm 0.46$ \\
\hline 6 & -7.64 & $-5.64 \pm 0.30$ & $-7.79 \pm 0.19$ & $-9.36 \pm 0.41$ & $-7.77 \pm 0.12$ \\
\hline 7 & -7.50 & $-6.99 \pm 0.25$ & $-8.14 \pm 0.18$ & $-8.98 \pm 0.33$ & $-8.14 \pm 0.13$ \\
\hline 8 & -8.41 & $-6.46 \pm 0.23$ & $-5.17 \pm 0.20$ & $-4.58 \pm 0.31$ & $-5.57 \pm 0.12$ \\
\hline 9 & $>-6.82$ & $-5.98 \pm 1.05$ & $-4.30 \pm 0.28$ & $-3.52 \pm 0.36$ & $-4.25 \pm 0.61$ \\
\hline 10 & -7.81 & $-6.47 \pm 0.23$ & $-6.31 \pm 0.16$ & $-5.37 \pm 0.31$ & $-6.04 \pm 0.12$ \\
\hline 11 & -10.22 & $-11.45 \pm 0.25$ & $-10.34 \pm 0.17$ & $-8.62 \pm 0.31$ & $-9.68 \pm 0.12$ \\
\hline 12 & -10.06 & $-11.50 \pm 0.29$ & $-10.51 \pm 0.16$ & $-8.90 \pm 0.31$ & $-10.06 \pm 0.13$ \\
\hline 13 & -10.71 & $-11.01 \pm 0.28$ & $-11.04 \pm 0.17$ & $-9.44 \pm 0.31$ & $-10.32 \pm 0.12$ \\
\hline 14 & -10.60 & $-10.16 \pm 0.24$ & $-9.88 \pm 0.16$ & $-9.05 \pm 0.31$ & $-10.06 \pm 0.13$ \\
\hline 15 & -9.83 & $-10.43 \pm 0.23$ & $-10.01 \pm 0.16$ & $-8.87 \pm 0.31$ & $-10.50 \pm 0.12$ \\
\hline 16 & -10.30 & $-13.56 \pm 0.23$ & $-12.35 \pm 0.16$ & $-11.54 \pm 0.32$ & $-12.33 \pm 0.12$ \\
\hline 17 & -10.45 & $-10.67 \pm 0.25$ & $-10.58 \pm 0.16$ & $-9.20 \pm 0.31$ & $-10.04 \pm 0.12$ \\
\hline 18 & -10.01 & $-10.53 \pm 0.24$ & $-10.38 \pm 0.17$ & $-9.32 \pm 0.31$ & $-9.87 \pm 0.15$ \\
\hline 19 & -10.30 & $-10.06 \pm 0.24$ & $-9.57 \pm 0.16$ & $-8.73 \pm 0.31$ & $-9.63 \pm 0.12$ \\
\hline 20 & -10.30 & $-9.08 \pm 0.24$ & $-8.14 \pm 0.17$ & $-6.79 \pm 0.31$ & $-8.02 \pm 0.12$ \\
\hline 21 & -10.37 & $-10.74 \pm 0.23$ & $-10.11 \pm 0.16$ & $-9.17 \pm 0.31$ & $-9.74 \pm 0.13$ \\
\hline 22 & -10.45 & $-10.42 \pm 0.23$ & $-9.39 \pm 0.16$ & $-8.36 \pm 0.31$ & $-9.12 \pm 0.12$ \\
\hline 23 & -10.14 & $-11.43 \pm 0.24$ & $-10.54 \pm 0.16$ & $-9.75 \pm 0.31$ & $-10.78 \pm 0.12$ \\
\hline 24 & $>-6.82$ & $-3.21 \pm 0.59$ & $-2.37 \pm 0.24$ & & \\
\hline 25 & -8.41 & $-7.49 \pm 0.25$ & $-6.12 \pm 0.16$ & $-5.63 \pm 0.31$ & $-6.65 \pm 0.13$ \\
\hline 26 & $>-6.82$ & $-4.76 \pm 0.69$ & $-3.68 \pm 0.24$ & & $-2.84 \pm 0.67$ \\
\hline 27 & -8.74 & $-9.67 \pm 0.29$ & $-8.46 \pm 0.16$ & $-6.48 \pm 0.32$ & $-8.10 \pm 0.14$ \\
\hline 28 & -10.06 & $-9.19 \pm 0.23$ & $-8.98 \pm 0.16$ & $-7.47 \pm 0.31$ & $-8.33 \pm 0.13$ \\
\hline 29 & -10.16 & $-12.15 \pm 0.36$ & $-12.66 \pm 0.19$ & $-14.20 \pm 0.49$ & \\
\hline 30 & -10.50 & $-9.33 \pm 0.26$ & $-10.01 \pm 0.18$ & $-10.87 \pm 0.33$ & $-10.21 \pm 0.13$ \\
\hline 31 & -11.32 & $-11.50 \pm 0.26$ & $-12.14 \pm 0.18$ & $-13.10 \pm 0.33$ & $-12.35 \pm 0.13$ \\
\hline 32 & -11.22 & $-12.26 \pm 0.25$ & $-13.81 \pm 0.19$ & $-13.88 \pm 0.33$ & $-13.08 \pm 0.13$ \\
\hline 33 & -11.63 & $-12.17 \pm 0.25$ & $-13.61 \pm 0.19$ & $-14.48 \pm 0.33$ & $-13.66 \pm 0.14$ \\
\hline 34 & -10.19 & $-9.89 \pm 0.25$ & $-10.71 \pm 0.19$ & $-11.38 \pm 0.33$ & $-10.70 \pm 0.13$ \\
\hline 35 & -9.89 & $-9.16 \pm 0.25$ & $-9.85 \pm 0.18$ & $-10.65 \pm 0.33$ & $-9.96 \pm 0.14$ \\
\hline 36 & -9.20 & $-8.53 \pm 0.25$ & & $-10.60 \pm 0.33$ & $-9.88 \pm 0.14$ \\
\hline 37 & -9.59 & $-10.19 \pm 0.27$ & $-10.69 \pm 0.18$ & $-11.67 \pm 0.33$ & $-10.80 \pm 0.13$ \\
\hline 38 & -10.71 & $-10.61 \pm 0.25$ & & $-12.43 \pm 0.33$ & $-11.62 \pm 0.14$ \\
\hline 39 & -10.91 & $-11.39 \pm 0.25$ & & $-13.18 \pm 0.36$ & $-12.41 \pm 0.14$ \\
\hline 40 & -10.50 & $-10.35 \pm 0.25$ & & $-12.40 \pm 0.35$ & $-11.32 \pm 0.14$ \\
\hline 41 & -10.09 & & & $-12.39 \pm 0.34$ & $-11.69 \pm 0.14$ \\
\hline
\end{tabular}

${ }^{a}$ All $\Delta G$ values and predicted errors are given in $\mathrm{kcal} \cdot \mathrm{mol}^{-1}$. 
Table S8: Experimental Binding Affinity and FEP+ Predictions for the HSP90 Data Set with 20 ns Simulation Time per $\lambda$ Window. ${ }^{a}$

\begin{tabular}{|c|c|c|c|c|c|}
\hline Ligand & $\Delta G_{\text {exp }}$ & $\Delta G_{\text {mcsdocking }}$ & $\Delta G_{\text {SPcore01 }}$ & $\Delta G_{\text {flex_scaf }}$ & $\Delta G_{\text {flex_mcs }}$ \\
\hline 1 & -11.63 & $-11.85 \pm 0.18$ & $-13.19 \pm 0.16$ & $-13.45 \pm 0.33$ & $-13.13 \pm 0.14$ \\
\hline 3 & -9.06 & $-6.1 \pm 0.18$ & $-7.80 \pm 0.16$ & $-7.53 \pm 0.31$ & $-7.20 \pm 0.14$ \\
\hline $4-0$ & $>-6.82$ & $-4.63 \pm 0.36$ & $-6.57 \pm 0.72$ & $-3.48 \pm 2.24$ & $-6.24 \pm 0.29$ \\
\hline $4-1$ & $>-6.82$ & & $-2.27 \pm 0.43$ & & \\
\hline 5 & $>-6.82$ & $-6.89 \pm 0.40$ & $-9.17 \pm 0.67$ & $-9.18 \pm 0.36$ & $-8.93 \pm 0.46$ \\
\hline 6 & -7.64 & $-5.99 \pm 0.18$ & $-7.95 \pm 0.16$ & $-8.74 \pm 0.39$ & $-7.76 \pm 0.13$ \\
\hline 7 & -7.50 & $-6.61 \pm 0.17$ & $-8.73 \pm 0.17$ & $-8.44 \pm 0.31$ & $-8.13 \pm 0.13$ \\
\hline 8 & -8.41 & $-6.64 \pm 0.17$ & $-5.19 \pm 0.15$ & $-5.11 \pm 0.30$ & $-5.57 \pm 0.12$ \\
\hline 9 & $>-6.82$ & $-6.15 \pm 1.00$ & $-4.29 \pm 0.41$ & $-4.05 \pm 0.35$ & $-4.25 \pm 0.61$ \\
\hline 10 & -7.81 & $-6.65 \pm 0.17$ & $-6.26 \pm 0.14$ & $-5.91 \pm 0.30$ & $-6.05 \pm 0.12$ \\
\hline 11 & -10.22 & $-11.62 \pm 0.20$ & $-10.00 \pm 0.15$ & $-9.16 \pm 0.30$ & $-9.68 \pm 0.12$ \\
\hline 12 & -10.06 & $-11.67 \pm 0.24$ & $-10.39 \pm 0.14$ & $-9.44 \pm 0.30$ & $-10.07 \pm 0.13$ \\
\hline 13 & -10.71 & $-11.19 \pm 0.23$ & $-10.70 \pm 0.15$ & $-9.98 \pm 0.30$ & $-10.32 \pm 0.12$ \\
\hline 14 & -10.60 & $-10.33 \pm 0.17$ & $-9.60 \pm 0.15$ & $-9.59 \pm 0.30$ & $-10.07 \pm 0.13$ \\
\hline 15 & -9.83 & $-10.61 \pm 0.17$ & $-9.82 \pm 0.15$ & $-9.40 \pm 0.30$ & $-10.50 \pm 0.12$ \\
\hline 16 & -10.30 & $-13.74 \pm 0.17$ & $-12.15 \pm 0.15$ & $-12.08 \pm 0.31$ & $-12.34 \pm 0.12$ \\
\hline 17 & -10.45 & $-10.84 \pm 0.19$ & $-10.23 \pm 0.14$ & $-9.74 \pm 0.30$ & $-10.04 \pm 0.12$ \\
\hline 18 & -10.01 & $-10.71 \pm 0.18$ & $-9.84 \pm 0.14$ & $-9.86 \pm 0.30$ & $-9.88 \pm 0.16$ \\
\hline 19 & -10.30 & $-10.24 \pm 0.18$ & $-9.42 \pm 0.14$ & $-9.26 \pm 0.30$ & $-9.63 \pm 0.12$ \\
\hline 20 & -10.30 & $-9.26 \pm 0.17$ & $-7.69 \pm 0.15$ & $-7.32 \pm 0.30$ & $-8.03 \pm 0.13$ \\
\hline 21 & -10.37 & $-10.92 \pm 0.17$ & $-9.92 \pm 0.14$ & $-9.70 \pm 0.30$ & $-9.75 \pm 0.13$ \\
\hline 22 & -10.45 & $-10.60 \pm 0.17$ & $-9.26 \pm 0.14$ & $-8.90 \pm 0.30$ & $-9.13 \pm 0.12$ \\
\hline 23 & -10.14 & $-11.60 \pm 0.18$ & $-10.37 \pm 0.14$ & $-10.28 \pm 0.30$ & $-10.79 \pm 0.12$ \\
\hline 24 & $>-6.82$ & $-3.38 \pm 0.56$ & $-2.15 \pm 0.24$ & & \\
\hline 25 & -8.41 & $-7.67 \pm 0.19$ & $-5.94 \pm 0.14$ & $-6.16 \pm 0.30$ & $-6.65 \pm 0.13$ \\
\hline 26 & $>-6.82$ & $-4.93 \pm 0.64$ & $-3.43 \pm 0.24$ & & $-3.79 \pm 0.67$ \\
\hline 27 & -8.74 & $-9.83 \pm 0.24$ & $-7.99 \pm 0.16$ & $-7.02 \pm 0.31$ & $-8.10 \pm 0.15$ \\
\hline 28 & -10.06 & $-9.37 \pm 0.17$ & $-8.72 \pm 0.14$ & $-8.00 \pm 0.30$ & $-8.33 \pm 0.13$ \\
\hline 29 & -10.16 & $-12.30 \pm 0.19$ & $-12.96 \pm 0.17$ & $-13.63 \pm 0.47$ & \\
\hline 30 & -10.50 & $-9.07 \pm 0.18$ & $-10.50 \pm 0.17$ & $-10.30 \pm 0.32$ & $-10.20 \pm 0.14$ \\
\hline 31 & -11.32 & $-11.22 \pm 0.19$ & $-12.51 \pm 0.16$ & $-12.55 \pm 0.31$ & $-12.35 \pm 0.14$ \\
\hline 32 & -11.22 & $-11.99 \pm 0.18$ & $-14.08 \pm 0.16$ & $-13.31 \pm 0.32$ & $-13.07 \pm 0.14$ \\
\hline 33 & -11.63 & $-11.90 \pm 0.18$ & $-13.83 \pm 0.16$ & $-13.92 \pm 0.32$ & $-13.66 \pm 0.14$ \\
\hline 34 & -10.19 & $-9.66 \pm 0.18$ & $-11.04 \pm 0.16$ & $-10.82 \pm 0.32$ & $-10.70 \pm 0.13$ \\
\hline 35 & -9.89 & $-9.04 \pm 0.18$ & $-10.28 \pm 0.17$ & $-10.09 \pm 0.32$ & $-9.96 \pm 0.14$ \\
\hline 36 & -9.20 & $-8.21 \pm 0.18$ & & $-10.04 \pm 0.32$ & $-9.87 \pm 0.14$ \\
\hline 37 & -9.59 & $-9.92 \pm 0.19$ & $-11.14 \pm 0.17$ & $-11.09 \pm 0.32$ & $-10.80 \pm 0.13$ \\
\hline 38 & -10.71 & $-10.32 \pm 0.18$ & & $-11.85 \pm 0.32$ & $-11.61 \pm 0.14$ \\
\hline 39 & -10.91 & $-11.13 \pm 0.18$ & & $-12.62 \pm 0.34$ & $-12.41 \pm 0.14$ \\
\hline 40 & -10.50 & $-10.06 \pm 0.18$ & & $-11.84 \pm 0.33$ & $-11.31 \pm 0.14$ \\
\hline 41 & -10.09 & & & $-11.82 \pm 0.32$ & $-11.69 \pm 0.14$ \\
\hline
\end{tabular}

${ }^{a}$ All $\Delta G$ values and predicted errors are given in $\mathrm{kcal} \cdot \mathrm{mol}^{-1}$. 
Table S9: Experimental Binding Affinity and FEP+ Predictions for the HNE Data Set. ${ }^{a}$

\begin{tabular}{lrrrr}
\hline Ligand & $\Delta G_{\text {exp }}$ & $\Delta G_{\text {mcsdocking }}$ & $\Delta G_{\text {SPcore01 }}$ & $\Delta G_{\text {flex_smarts }}$ \\
\hline 6 & -7.03 & $-2.59 \pm 0.18$ & & $-3.49 \pm 0.16$ \\
14 & -10.33 & $-11.70 \pm 0.14$ & $-11.20 \pm 0.16$ & $-11.72 \pm 0.16$ \\
15 & -12.28 & $-12.16 \pm 0.14$ & $-11.68 \pm 0.15$ & $-12.22 \pm 0.15$ \\
16 & -11.84 & $-10.69 \pm 0.14$ & $-10.42 \pm 0.16$ & $-10.96 \pm 0.14$ \\
17 & -11.41 & $-11.42 \pm 0.15$ & $-11.10 \pm 0.16$ & $-11.70 \pm 0.15$ \\
18 & -10.76 & $-11.21 \pm 0.14$ & $-10.79 \pm 0.15$ & $-11.41 \pm 0.14$ \\
19 & -11.54 & $-10.18 \pm 0.14$ & & $-10.27 \pm 0.14$ \\
20 & -10.50 & $-12.36 \pm 0.14$ & $-12.16 \pm 0.16$ & $-12.70 \pm 0.14$ \\
21 & -12.28 & $-11.54 \pm 0.16$ & $-11.32 \pm 0.16$ & $-12.79 \pm 0.15$ \\
22 & -11.36 & $-10.66 \pm 0.13$ & $-10.68 \pm 0.15$ & $-10.57 \pm 0.15$ \\
23 & -10.37 & $-12.47 \pm 0.14$ & $-11.75 \pm 0.15$ & $-12.03 \pm 0.15$ \\
$24-0$ & -11.32 & & & $-11.76 \pm 0.16$ \\
$24-1$ & -11.32 & & & $-11.63 \pm 0.25$ \\
25 & -12.00 & $-12.19 \pm 0.14$ & & $-11.65 \pm 0.18$ \\
26 & -12.58 & $-12.64 \pm 0.16$ & $-12.61 \pm 0.15$ & $-12.31 \pm 0.19$ \\
27 & -14.49 & $-14.53 \pm 0.15$ & & $-14.19 \pm 0.17$ \\
28 & -12.64 & $-12.25 \pm 0.15$ & & $-11.59 \pm 0.21$ \\
29 & -13.90 & $-15.81 \pm 0.14$ & & $-14.25 \pm 0.17$ \\
30 & -13.10 & $-14.01 \pm 0.14$ & & $-14.12 \pm 0.20$ \\
\hline 0
\end{tabular}

${ }^{a}$ All $\Delta G$ values and predicted errors are given in $\mathrm{kcal} \cdot \mathrm{mol}^{-1}$.

Table S10: Experimental Binding Affinity and FEP+ Predictions for the HNE Data Set with 20 ns Simulation Time per $\lambda$ Window. ${ }^{a}$

\begin{tabular}{lrrr}
\hline Ligand & $\Delta G_{\text {exp }}$ & $\Delta G_{\text {mcsdocking }}$ & $\Delta G_{\text {SPcore } 1}$ \\
\hline 6 & -7.03 & $-2.60 \pm 0.12$ & \\
14 & -10.33 & $-11.45 \pm 0.11$ & $-11.15 \pm 0.13$ \\
15 & -12.28 & $-12.08 \pm 0.11$ & $-11.55 \pm 0.13$ \\
16 & -11.84 & $-10.60 \pm 0.12$ & $-10.27 \pm 0.13$ \\
17 & -11.41 & $-11.42 \pm 0.11$ & $-11.10 \pm 0.13$ \\
18 & -10.76 & $-11.25 \pm 0.12$ & $-10.83 \pm 0.13$ \\
19 & -11.54 & $-9.89 \pm 0.12$ & $-12.06 \pm 0.13$ \\
20 & -10.50 & $-12.36 \pm 0.12$ & $-11.64 \pm 0.13$ \\
21 & -12.28 & $-11.85 \pm 0.12$ & $-10.57 \pm 0.13$ \\
22 & -11.36 & $-10.60 \pm 0.11$ & $-11.84 \pm 0.13$ \\
23 & -10.37 & $-12.60 \pm 0.12$ & \\
25 & -12.00 & $-12.32 \pm 0.11$ & \\
26 & -12.58 & $-12.59 \pm 0.12$ & $-12.70 \pm 0.13$ \\
27 & -14.49 & $-14.36 \pm 0.12$ & \\
28 & -12.64 & $-12.46 \pm 0.12$ & \\
29 & -13.90 & $-15.88 \pm 0.12$ & \\
30 & -13.10 & $-14.11 \pm 0.13$ & \\
\hline
\end{tabular}

${ }^{a}$ All $\Delta G$ values and predicted errors are given in $\mathrm{kcal} \cdot \mathrm{mol}^{-1}$. 
Table S11: Experimental Binding Affinity and FEP+ Predictions for the eg5 Data Set. ${ }^{a}$

\begin{tabular}{|c|c|c|c|c|c|}
\hline Ligand & $\Delta G_{\exp }$ & $\Delta G_{\text {mcsdocking }}$ & $\Delta G_{\text {SPcore01 }}$ & $\Delta G_{\text {flex_scaf }}$ & $\Delta G_{\text {flex_mcs }}$ \\
\hline CHEMBL1077204 & -11.05 & $-12.31 \pm 0.33$ & $-11.91 \pm 0.26$ & $-13.30 \pm 0.34$ & $-12.79 \pm 0.30$ \\
\hline CHEMBL1077227 & -11.32 & $-10.35 \pm 0.22$ & $-10.35 \pm 0.20$ & $-10.39 \pm 0.25$ & $-9.95 \pm 0.30$ \\
\hline CHEMBL1078691 & -10.19 & $-10.45 \pm 0.24$ & $-10.97 \pm 0.21$ & $-10.60 \pm 0.28$ & $-9.69 \pm 0.28$ \\
\hline CHEMBL1078774 & -10.60 & $-11.14 \pm 0.24$ & $-11.34 \pm 0.19$ & $-10.05 \pm 0.29$ & $-8.63 \pm 0.37$ \\
\hline CHEMBL1078998 & -10.37 & $-10.49 \pm 0.22$ & $-10.50 \pm 0.20$ & $-9.49 \pm 0.25$ & $-9.88 \pm 0.29$ \\
\hline CHEMBL1082248 & -11.63 & $-11.66 \pm 0.27$ & $-11.08 \pm 0.18$ & -10.93 & -11.1 \\
\hline CHEMBL1082249 & -11.22 & $-11.01 \pm 0.24$ & $-11.36 \pm 0.20$ & $-11.83 \pm 0.23$ & $-12.93 \pm 0.33$ \\
\hline CHEMBL1083517 & -11.46 & $-11.29 \pm 0.30$ & $-10.70 \pm 0.19$ & $-11.11 \pm 0.22$ & $-10.95 \pm 0.29$ \\
\hline CHEMBL1083836 & -10.86 & $-10.01 \pm 0.25$ & $-10.68 \pm 0.20$ & $-10.45 \pm 0.23$ & $-11.08 \pm 0.33$ \\
\hline CHEMBL1084115 & -9.44 & $-9.89 \pm 0.23$ & $-10.43 \pm 0.21$ & $-9.42 \pm 0.28$ & $-9.27 \pm 0.27$ \\
\hline CHEMBL1084143 & -11.87 & $-11.55 \pm 0.24$ & $-12.11 \pm 0.18$ & -12.60 & -13.4 \\
\hline CHEMBL1084431 & -9.80 & $-9.56 \pm 0.21$ & & $-12.17 \pm 0.30$ & $-10.35 \pm 0.30$ \\
\hline CHEMBL1084676 & -10.71 & $-11.54 \pm 0.21$ & $-10.20 \pm 0.22$ & $-12.24 \pm 0.32$ & $-11.28 \pm 0.28$ \\
\hline CHEMBL1084677 & -11.46 & $-12.06 \pm 0.24$ & $-12.46 \pm 0.20$ & $-11.79 \pm 0.24$ & $-13.73 \pm 0.35$ \\
\hline CHEMBL1084678 & -11.13 & $-11.21 \pm 0.26$ & $-11.98 \pm 0.28$ & $-11.57 \pm 0.32$ & $-12.56 \pm 0.33$ \\
\hline CHEMBL1084935-0 & -10.53 & $-7.80 \pm 0.57$ & $-9.54 \pm 0.22$ & $-8.14 \pm 0.27$ & $-8.10 \pm 0.38$ \\
\hline CHEMBL1084935-1 & -10.53 & & $-8.30 \pm 0.49$ & $-8.05 \pm 0.82$ & $-8.56 \pm 0.28$ \\
\hline CHEMBL1085666 & -10.50 & $-9.56 \pm 0.27$ & $-10.31 \pm 0.17$ & $-9.64 \pm 0.27$ & $-10.31 \pm 0.29$ \\
\hline CHEMBL1085692 & -11.32 & $-12.84 \pm 0.25$ & $-12.15 \pm 0.22$ & $-13.53 \pm 0.28$ & $-13.74 \pm 0.29$ \\
\hline CHEMBL1085895 & -10.86 & $-11.31 \pm 0.27$ & $-11.63 \pm 0.21$ & $-10.43 \pm 0.26$ & $-10.67 \pm 0.34$ \\
\hline CHEMBL1086409 & -9.87 & $-9.50 \pm 0.21$ & $-9.27 \pm 0.20$ & $-9.94 \pm 0.22$ & $-9.66 \pm 0.28$ \\
\hline CHEMBL1086410 & -11.46 & $-9.93 \pm 0.21$ & $-10.04 \pm 0.18$ & $-9.99 \pm 0.23$ & $-9.68 \pm 0.27$ \\
\hline CHEMBL1088740 & -8.70 & $-8.26 \pm 0.25$ & $-8.45 \pm 0.20$ & $-8.06 \pm 0.28$ & $-8.05 \pm 0.30$ \\
\hline CHEMBL1089056 & -9.44 & $-9.29 \pm 0.22$ & $-9.74 \pm 0.18$ & $-9.14 \pm 0.24$ & $-9.11 \pm 0.27$ \\
\hline CHEMBL1089393 & -8.38 & $-7.65 \pm 0.25$ & $-8.69 \pm 0.19$ & $-8.06 \pm 0.25$ & $-8.01 \pm 0.28$ \\
\hline CHEMBL1093087 & -10.53 & $-13.34 \pm 0.28$ & & $-11.36 \pm 0.30$ & $-12.19 \pm 0.39$ \\
\hline CHEMBL1093088 & -11.22 & $-8.70 \pm 0.26$ & $-8.73 \pm 0.19$ & $-8.62 \pm 0.31$ & $-7.69 \pm 0.33$ \\
\hline CHEMBL1096002 & -11.13 & $-11.49 \pm 0.22$ & $-11.54 \pm 0.24$ & $-11.50 \pm 0.23$ & $-10.52 \pm 0.33$ \\
\hline CHEMBL1096003 & -11.87 & $-13.58 \pm 0.31$ & $-12.40 \pm 0.20$ & $-12.55 \pm 0.31$ & $-13.09 \pm 0.30$ \\
\hline
\end{tabular}

${ }^{a}$ All $\Delta G$ values and predicted errors are given in $\mathrm{kcal} \cdot \mathrm{mol}^{-1}$. 
Table S12: Experimental Binding Affinity and FEP+ Predictions for the eg5 Data Set with 20 ns Simulation Time per $\lambda$ Window. ${ }^{a}$

\begin{tabular}{|c|c|c|c|c|c|}
\hline Ligand & $\Delta G_{\exp }$ & $\Delta G_{\text {mcsdocking }}$ & $\Delta G_{\text {SPcore } 01}$ & $\Delta G_{\text {flex_scaf }}$ & $\Delta G_{\text {flex_mcs }}$ \\
\hline CHEMBL1077204 & -11 & $-12.11 \pm 0.19$ & $-12.11 \pm 0.17$ & $-12.86 \pm 0.27$ & $-12.41 \pm 0.29$ \\
\hline CHEMBL1077227 & -11.32 & $-10.36 \pm 0.17$ & $-10.34 \pm 0.18$ & $-10.42 \pm 0.18$ & $-9.95 \pm 0.22$ \\
\hline CHEMBL1078691 & -10.19 & $-10.62 \pm 0.18$ & $-10.95 \pm 0.19$ & $-10.48 \pm 0.23$ & $-9.78 \pm 0.20$ \\
\hline CHEMBL1078774 & -10.60 & $-10.98 \pm 0.18$ & $-11.33 \pm 0.18$ & $-9.98 \pm 0.25$ & $-8.85 \pm 0.18$ \\
\hline CHEMBL1078998 & -10.37 & $-10.47 \pm 0.17$ & $-10.48 \pm 0.18$ & $-9.49 \pm 0.19$ & $-10.03 \pm 0.19$ \\
\hline CHEMBL1082248 & -11.63 & $-11.73 \pm 0.17$ & $-10.99 \pm 0.16$ & $-10.96 \pm 0.19$ & $-10.99 \pm 0.22$ \\
\hline CHEMBL1082249 & -11.22 & $-10.77 \pm 0.18$ & $-8.71 \pm 0.17$ & $-8.55 \pm 0.26$ & $-8.38 \pm 0.18$ \\
\hline CHEMBL1083517 & -11.46 & $-11.45 \pm 0.19$ & $-10.69 \pm 0.17$ & $-11.09 \pm 0.17$ & $-10.58 \pm 0.19$ \\
\hline CHEMBL1083836 & -10.86 & $-9.86 \pm 0.18$ & $-10.33 \pm 0.18$ & $-10.36 \pm 0.20$ & $-10.82 \pm 0.21$ \\
\hline CHEMBL1084115 & -9.44 & $-9.96 \pm 0.17$ & $-10.41 \pm 0.19$ & $-9.37 \pm 0.23$ & $-9.40 \pm 0.19$ \\
\hline CHEMBL1084143 & -11.87 & $-11.68 \pm 0.19$ & $-12.33 \pm 0.19$ & $-12.44 \pm 0.21$ & $-12.85 \pm 0.24$ \\
\hline CHEMBL1084431 & -9.80 & $-9.98 \pm 0.21$ & & $-12.05 \pm 0.28$ & $-10.46 \pm 0.26$ \\
\hline CHEMBL1084676 & -10.71 & $-11.71 \pm 0.20$ & $-10.61 \pm 0.17$ & $-12.21 \pm 0.19$ & $-11.03 \pm 0.21$ \\
\hline CHEMBL1084677 & -11.46 & $-12.33 \pm 0.19$ & $-10.01 \pm 0.16$ & $-10.08 \pm 0.17$ & \\
\hline CHEMBL1084678 & -11.13 & $-11.34 \pm 0.18$ & $-11.38 \pm 0.19$ & $-11.38 \pm 0.20$ & $-10.97 \pm 0.19$ \\
\hline CHEMBL1084935-0 & -10.53 & $-8.89 \pm 0.21$ & $-9.53 \pm 0.21$ & $-8.43 \pm 0.20$ & $-8.47 \pm 0.19$ \\
\hline CHEMBL1084935-1 & -10.53 & $-7.78 \pm 0.62$ & $-8.29 \pm 0.48$ & $-8.13 \pm 0.66$ & $-8.15 \pm 0.32$ \\
\hline CHEMBL1085666 & -10.50 & $-9.61 \pm 0.19$ & $-10.27 \pm 0.16$ & $-9.84 \pm 0.18$ & $-10.39 \pm 0.21$ \\
\hline CHEMBL1085692 & -11.32 & $-12.78 \pm 0.17$ & $-12.02 \pm 0.20$ & $-13.28 \pm 0.20$ & $-13.42 \pm 0.20$ \\
\hline CHEMBL1085895 & -10.86 & $-11.24 \pm 0.23$ & $-11.61 \pm 0.20$ & $-10.40 \pm 0.20$ & $-10.61 \pm 0.18$ \\
\hline CHEMBL1086409 & -9.87 & $-9.41 \pm 0.17$ & $-9.43 \pm 0.16$ & $-9.89 \pm 0.19$ & $-9.84 \pm 0.19$ \\
\hline CHEMBL1086410 & -11.46 & $-9.96 \pm 0.18$ & $-12.45 \pm 0.18$ & $-11.73 \pm 0.18$ & $-12.97 \pm 0.21$ \\
\hline CHEMBL1088740 & -8.70 & $-8.19 \pm 0.19$ & $-8.43 \pm 0.19$ & $-8.08 \pm 0.22$ & $-8.06 \pm 0.17$ \\
\hline CHEMBL1089056 & -9.44 & $-9.32 \pm 0.18$ & $-9.72 \pm 0.17$ & $-9.24 \pm 0.18$ & $-9.29 \pm 0.17$ \\
\hline CHEMBL1089393 & -8.38 & $-7.57 \pm 0.21$ & $-8.68 \pm 0.18$ & $-8.05 \pm 0.20$ & $-8.25 \pm 0.18$ \\
\hline CHEMBL1093087 & -10.53 & $-13.34 \pm 0.25$ & & $-11.56 \pm 0.20$ & $-12.88 \pm 0.20$ \\
\hline CHEMBL1093088 & -11.22 & $-8.66 \pm 0.23$ & $-11.27 \pm 0.18$ & $-11.79 \pm 0.19$ & $-12.48 \pm 0.21$ \\
\hline CHEMBL1096002 & -11.13 & $-11.56 \pm 0.18$ & $-12.44 \pm 0.18$ & $-12.12 \pm 0.19$ & $-12.85 \pm 0.21$ \\
\hline CHEMBL1096003 & -11.87 & $-13.04 \pm 0.20$ & $-12.04 \pm 0.16$ & $-12.78 \pm 0.18$ & $-13.24 \pm 0.23$ \\
\hline
\end{tabular}

${ }^{a}$ All $\Delta G$ values and predicted errors are given in $\mathrm{kcal} \cdot \mathrm{mol}^{-1}$. 


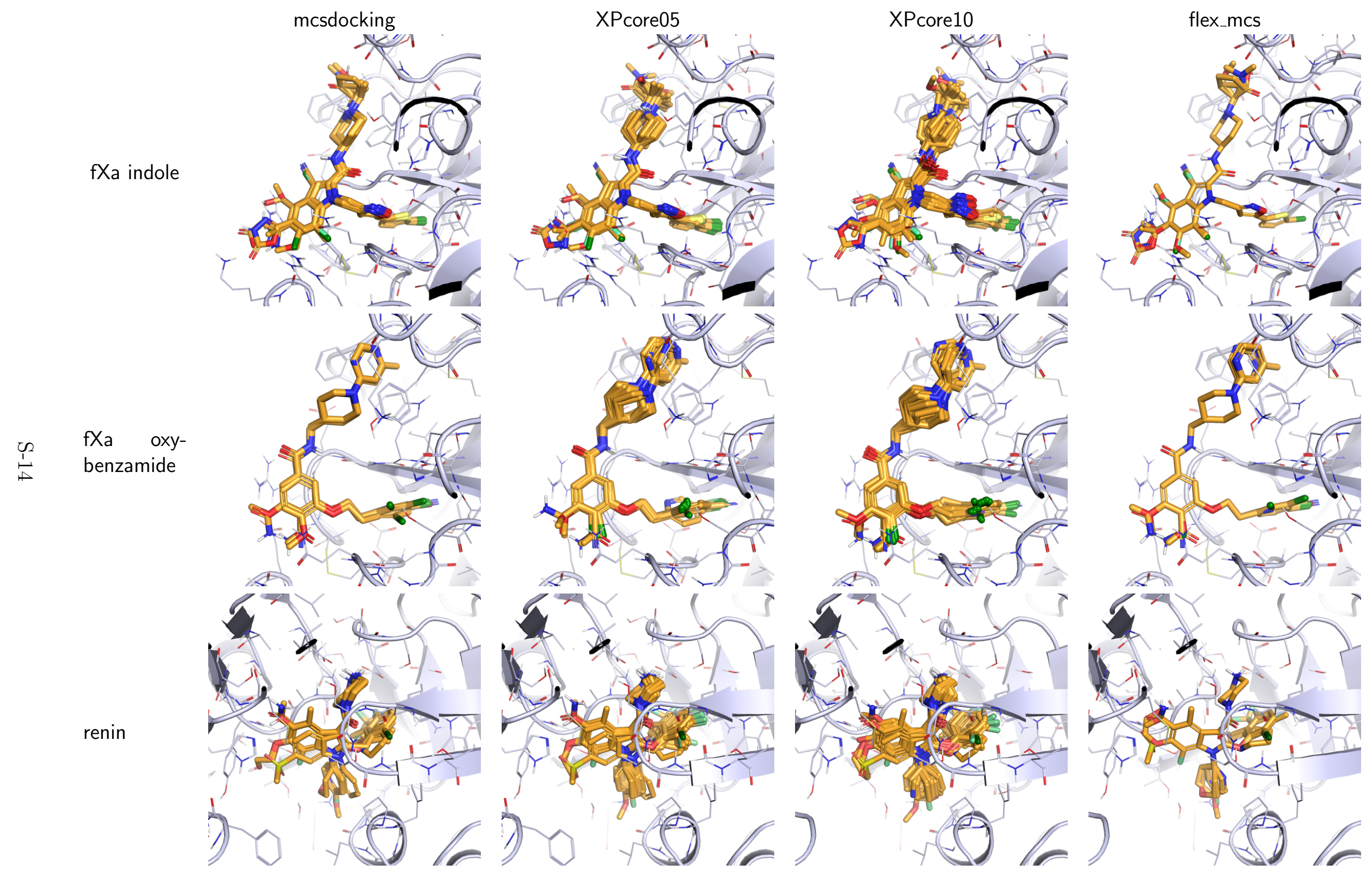

Figure S2: Poses generated by the different alignment approaches and used as input for FEP+ simulations for the two fXa ligand series as well as the renin data set. Images were generated using PyMOL. $\underline{\$ 1]}$ 


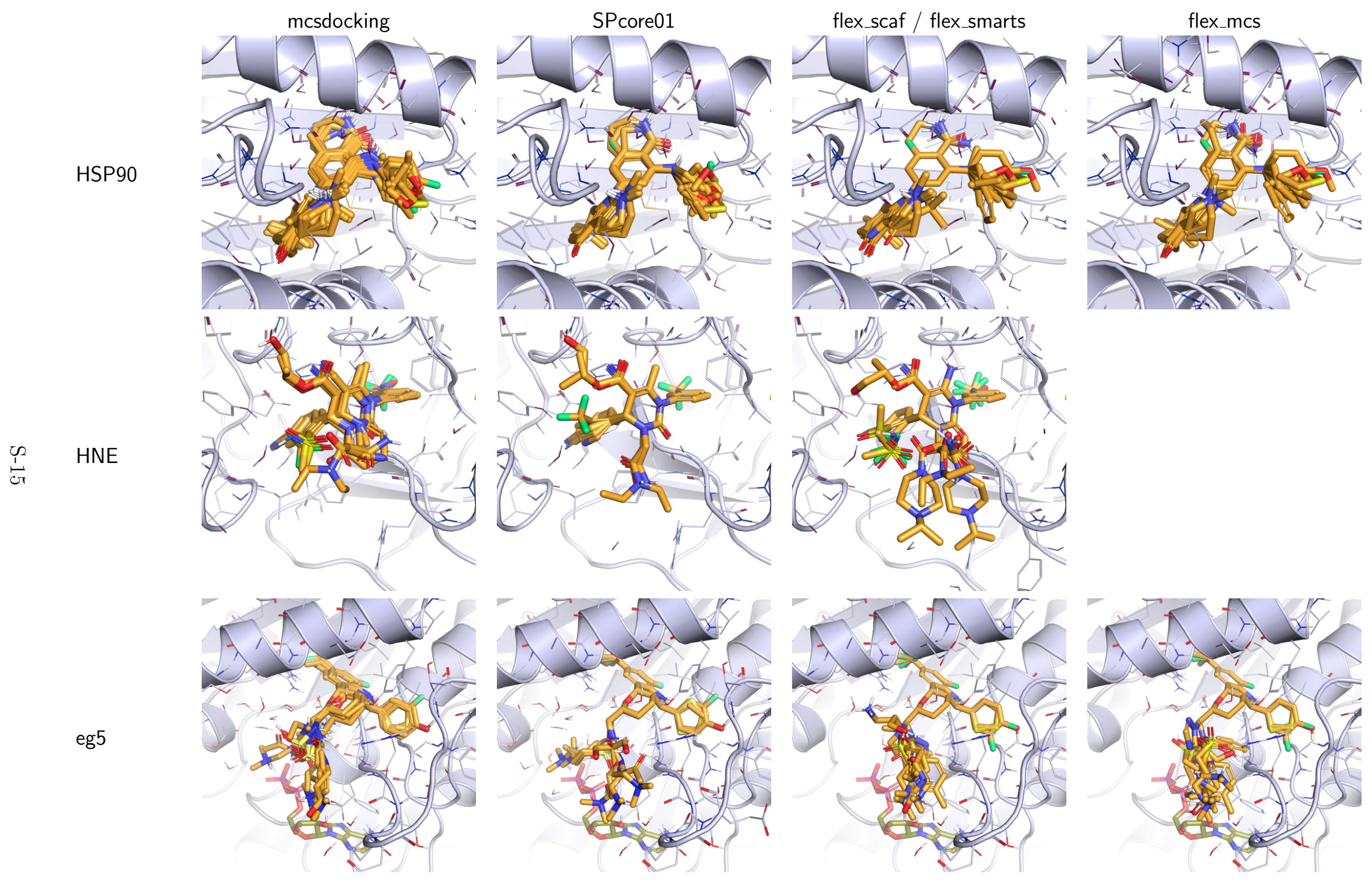

Figure S3: Poses generated by the different alignment approaches and used as input for FEP+ simulations for the HSP90, HNE and eg5 data set. For HNE flexible ligand alignment was performed with coordinate snapping for a pre-defined SMARTS for the core structure and not with automated MCS or Bemis-Murcko scaffold detection. Images were generated using PyMOL.\$1] 

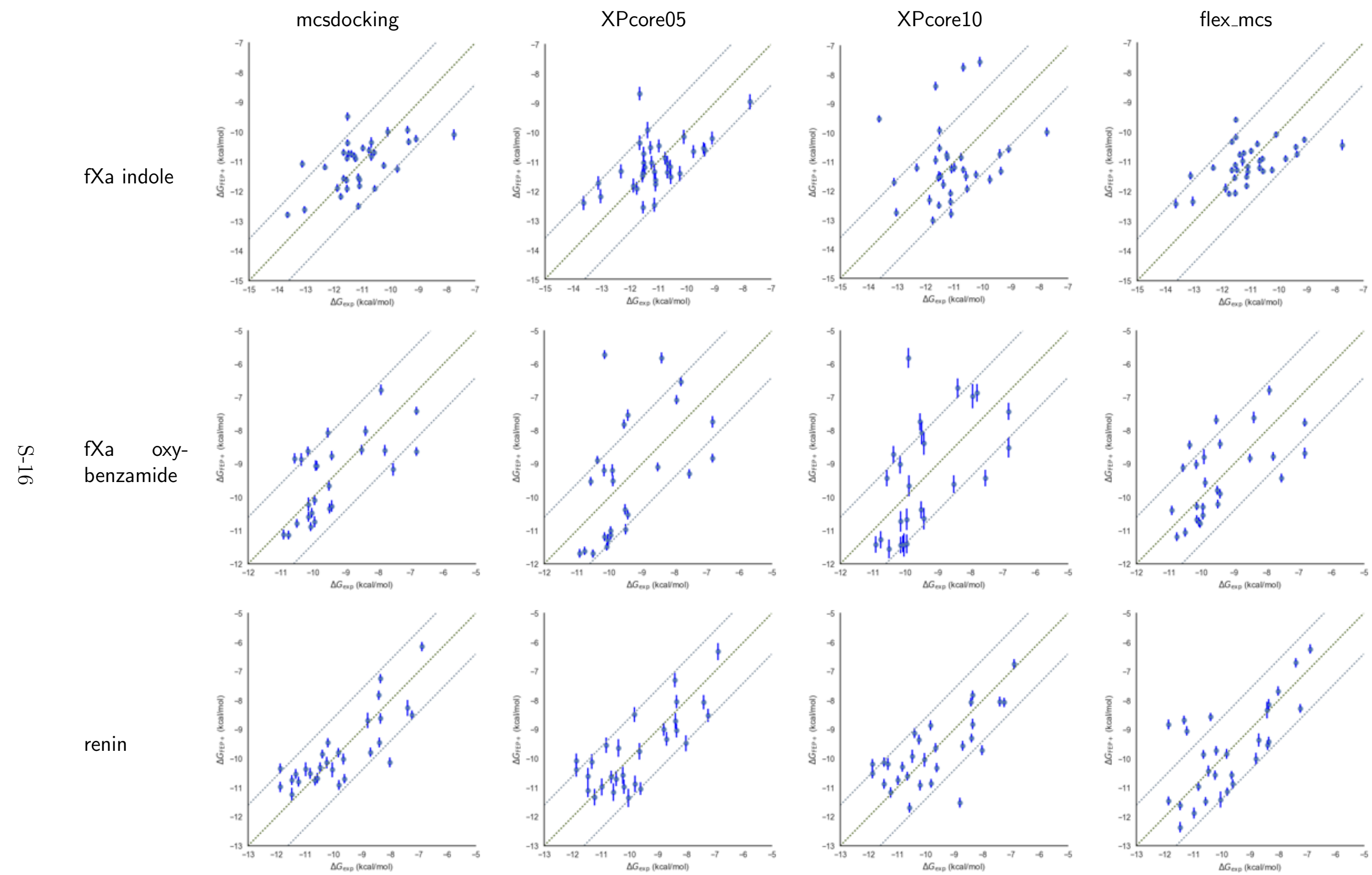

Figure S4: Correlation plots of $\Delta G$ (experiment against FEP+ predictions) for fXa ligand series and renin data set using different input ligand pose generation approaches. Dashed line at $\pm 1.4 \mathrm{kcal} / \mathrm{mol}$ shows $1 \mathrm{p} K_{\mathrm{i}} \log$ unit level for desirable prediction accuracy. 

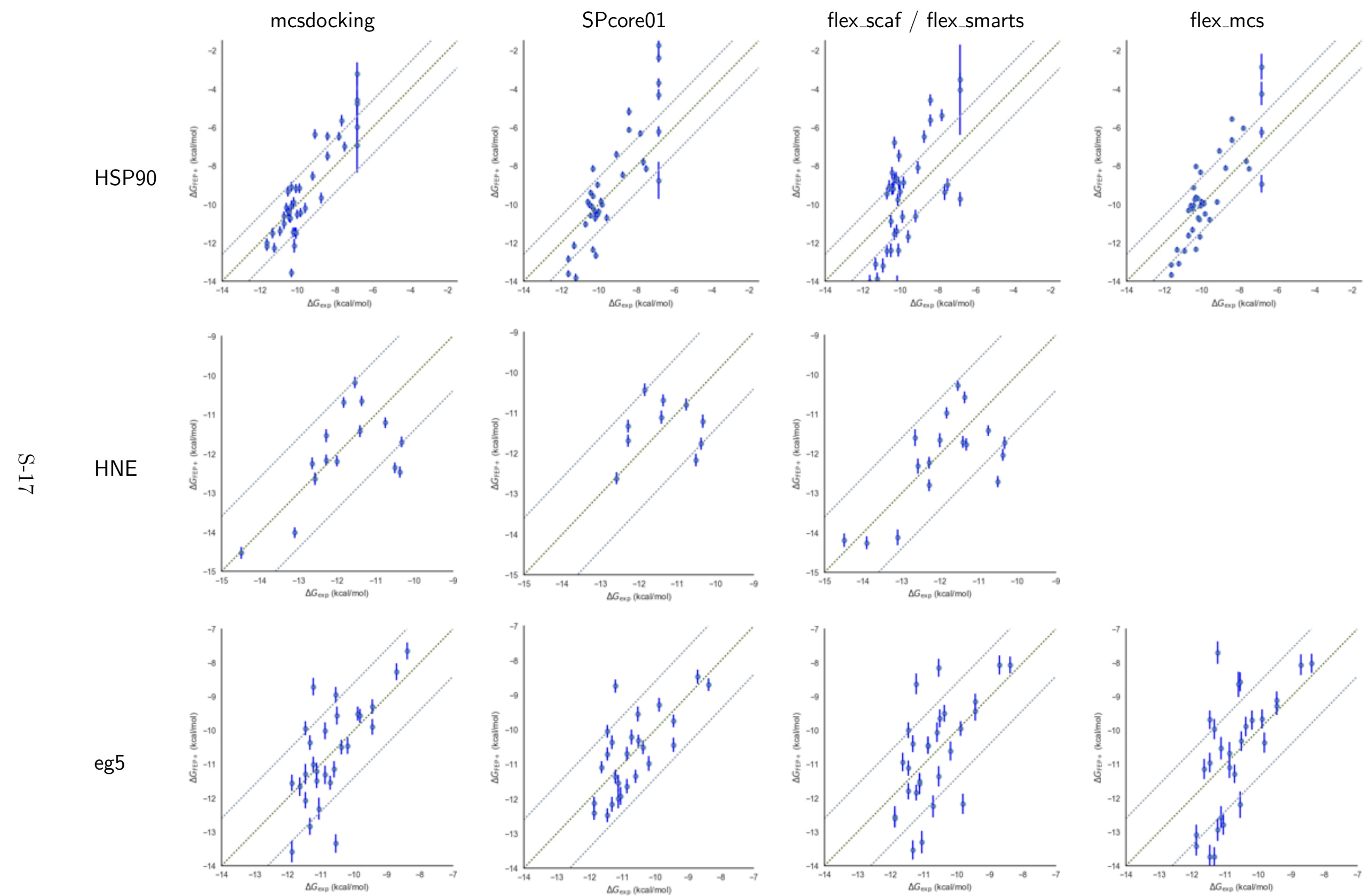

Figure S5: Correlation plots of experimental $\Delta G$ and corresponding prediction from FEP + using different input ligand pose generation approaches for the HSP90, HNE and eg5 data set. For HNE flexible ligand alignment was performed with coordinate snapping for a predefined SMARTS for the core structure and not with automated MCS or Bemis-Murcko scaffold detection. Dashed line at \pm 1.4 kcal/mol shows $1 \mathrm{p} K_{\mathrm{i}}$ log unit level for desirable prediction accuracy. 
Table S13: Pearson $R^{2}$ of FEP + Results from Different Ligand Alignments in Comparison with each other.

(a) fXa indole

\begin{tabular}{l|rrr}
\hline & XPcore05 & XPcore10 & flex_mcs \\
\hline mcsdocking & 0.42 & 0.19 & 0.84 \\
XPcore05 & & 0.28 & 0.45 \\
XPcore10 & & & 0.20 \\
\hline
\end{tabular}

(c) renin

\begin{tabular}{l|rrr}
\hline & XPcore05 & XPcore10 & flex_mcs \\
\hline mcsdocking & 0.77 & 0.62 & 0.55 \\
XPcore05 & & 0.71 & 0.56 \\
XPcore10 & & & 0.47 \\
\hline
\end{tabular}

(e) HNE

\begin{tabular}{l|rr}
\hline & SPcore01 & flex_smarts \\
\hline mcsdocking & 0.90 & 0.96 \\
SPcore01 & & 0.60 \\
\hline
\end{tabular}

(b) fXa oxybenzamide

\begin{tabular}{l|rrr}
\hline & XPcore05 & XPcore10 & flex_mcs \\
\hline mcsdocking & 0.58 & 0.67 & 0.93 \\
XPcore05 & & 0.51 & 0.60 \\
XPcore10 & & & 0.73 \\
\hline
\end{tabular}

(d) HSP90

\begin{tabular}{l|rrr}
\hline & SPcore01 & flex_scaf & flex_mcs \\
\hline mcsdocking & 0.85 & 0.51 & 0.78 \\
SPcore01 & & 0.86 & 0.98 \\
flex_scaf & & & 0.90 \\
\hline
\end{tabular}

(f) eg5

\begin{tabular}{l|rrr}
\hline & SPcore01 & flex_scaf & flex_mcs \\
\hline mcsdocking & 0.80 & 0.72 & 0.71 \\
SPcore01 & & 0.74 & 0.73 \\
flex_scaf & & & 0.81 \\
\hline
\end{tabular}



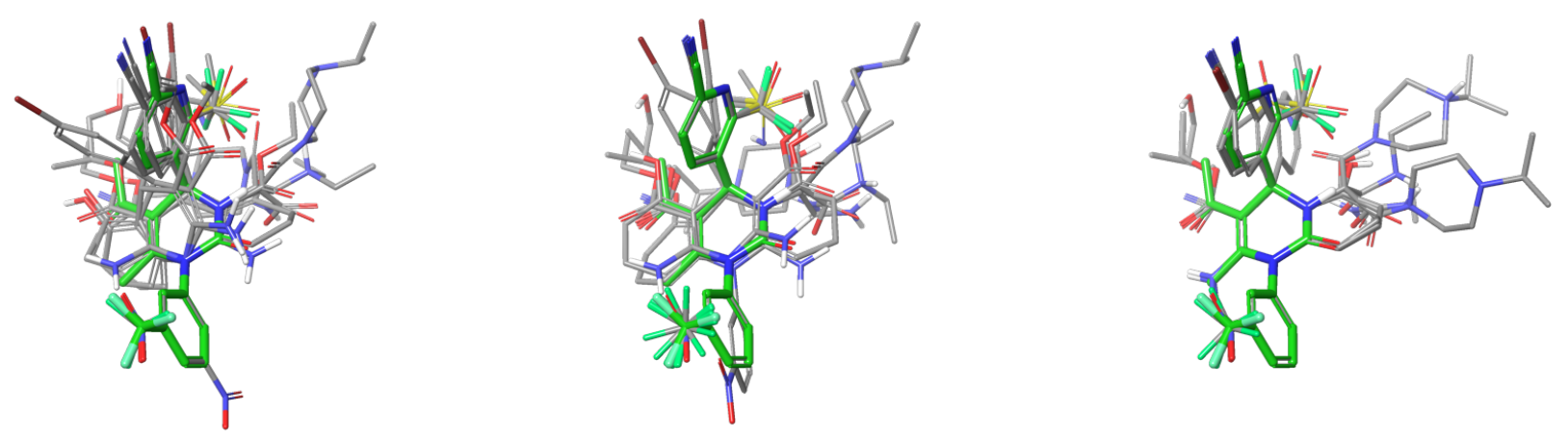

Figure S6: Poses for HNE generated using flexible ligand alignment with automatic detection of constrained common part using MCS (left), common scaffold (middle) and with manual specification of ligand core via SMARTS (right). The latter was used for RBFE simulations. Automatic detection of common part leads to constrained alignment of side chain instead of actual ligand series core.

\section{References}

(S1) Schrödinger, LLC, The PyMOL Molecular Graphics System, Version 2.3. 2019. 